\title{
Hesitant Fuzzy Multi-attribute Decision Making Method based on a New Entropy Measure
}

\author{
Zhengkun Yin \\ Changsha Vocational \& Technical College, Changsha 410010, China \\ chinayinzk@163.com
}

\begin{abstract}
Entropy measure is an important information measure of hesitant fuzzy sets. This article will construct a new entropy measure and then develop a new decision method for the multi-attribute decision making problem with attribute values expressed with hesitant fuzzy elements. Firstly, a new entropy measure of hesitant fuzzy sets is constructed, then the weights are obtained using the entropy weight method, and positive ideal solution and negative ideal solution are defined. Further, based on the conception of TOPSIS, the relative closeness degree is calculated to rank the alternatives. Finally, a practical example is examined to demonstrate the effectiveness and feasibility of the proposed method.
\end{abstract}

Keywords: hesitant fuzzy element, multi-attribute decision making, entropy measure, entropy weight method

\section{Introduction}

Fuzzy set [1] was firstly proposed by Zadeh in 1965, and because it can well describe the fuzzy concept of object, fuzzy set theory has been received great attention and applied to various fields. But fuzzy set is characterized by a single of membership degree function; it can only reflect the satisfied and unsatisfied situation. While in some cases, for example, for a vote decision problem, there are three values occur, which are support, against and abstain. In such a case, fuzzy sets can only reflect the degree support and against, which loses some uncertain information. Intuitionistic fuzzy set (IFS), introduced by Atanassov [2], is an extension of Zadeh's fuzzy set. An IFS considers the membership, nonmembership and hesitancy degree of three aspects of information. Thus IFSs can effectively depict the fuzziness, especially the 'neither this nor that' type of ambiguity. Atanassov and Gargov [3] further developed the IFS to the interval valued intuitionistic fuzzy set (IVIFS). More application of IFS and IVIFS in multiple attribute decision making fields can be seen in references, such as $\mathrm{Xu}$ [4], Li [5], and Wei [6]. Due to the ambiguity of the complexity of the objective world and human thinking, and the decisionmakers' knowledge background and knowledge of the different levels, in recent years, people found many decision problems often in disagreement situation, and thus lead the final decision showed hesitation or irresolute and hesitant state. For example, when several decision makers discuss the membership of, some decision makers thought is 0.5 , others thought to be 0.7 , they do not compromise with each other and then they cannot reach an agreement. In this situation, we can only express the membership of with the set $\{0.5,0.7\}$. Obviously this is different from the intuitionistic fuzzy membership of 0.4 with the expression as $\langle 0.4,0.4\rangle$. In order to better handle the multi-attribute decision making problem of this kind of situation, Torra and Narukawa [7,8] proposed an extended fuzzy set, called hesitant fuzzy set, to describe the decision maker's different opinions. In recent years, hesitant fuzzy set has attracted many scholars' attention and study. Xia and $\mathrm{Xu}$ [9] further defined the hesitant fuzzy set of algorithms, and proposed the hesitant fuzzy weighted average (HFWA) operator and hesitant fuzzy weighted geometric (HFWG) 
operators etc. $\mathrm{Xu}$ and $\mathrm{Xia}$ [10] defined hesitant fuzzy sets distance and similarity, and $\mathrm{Xu}$ and Zhang [11] considered the hesitant fuzzy multiple attribute decision making problems based on TOPSIS method; Zhang and Wei [12] studied the hesitant fuzzy multiple attribute decision making problems with VIKOR method and TOPSIS method. Entropy measure is an important measure for fuzzy set. Many methods have been proposed for constructing entropy measures for fuzzy sets and IFS, but little discussion is given for hesitant fuzzy set ([12-15]). In this paper, we shall develop a new entropy measure for IFSs and define the notions of positive ideal HFS and negative ideal HFS, and then apply the entropy measure to multiple attribute decision making based on hesitant fuzzy information.

The rest of this paper is organized as follows. In Section 2, the basic concepts and operations of hesitant fuzzy sets are firstly recalled, and then a new hesitant fuzzy entropy measure is constructed. In Section 3, a new decision making method is proposed based on the new hesitant fuzzy entropy measure. A practical example is given to demonstrate the feasibility and effectiveness of the proposed decision making method in Section 4. Finally, a conclusion is given in Section 5.

\section{Preliminary Knowledge}

The preliminary definitions and lemmas are given as below.

Torra and Narukawa [7] firstly introduced the concept of hesitant fuzzy sets (HFSs) as follows.

Definition $1[7,8]$. Let $X$ be a universe set, a hesitant fuzzy set $A$ in $X$ is in terms of a function that when applied to $X$ returns a subset of $[0,1]$, which can be represented as the following mathematical symbol:

$$
A=\left\{<x, h_{A}(x)>\mid x \in X\right\}
$$

where $h_{A}(x)$ is a set of some values in $[0,1]$, denoting the possible membership degrees of the element $x \in X$ to the set A. For convenience, Xia and Xu [10] named $h_{A}(x)$ a hesitant fuzzy element, and briefly denote it as HFE. Especially, if there is only one value in $h_{A}(x)$, then the hesitant fuzzy set reduces to the fuzzy set. That is to say fuzzy sets are a special type of hesitant fuzzy sets; therefore, the theory for hesitant fuzzy sets can also be applied to fuzzy sets.

It is noted that the number of values in different HFEs may be different, let $l(h)$ be the number of values in $h$. We can also find that the values in an HFE are out of order; we can arrange them in any order. For an HFE $h$, let $\sigma:(1,2, \ldots, n) \rightarrow(1,2, \ldots, n)$ be a permutation satisfying $h_{\sigma(j)} \leq h_{\sigma(j+1)}, j=1,2, \ldots, l(h)-1$.

Definition 2 [7, 8]. Given an HFE $\alpha$, the complement set of $\alpha$ is defined as

$$
\alpha^{c}=\bigcup_{\gamma \in \alpha}\left\{1-\gamma^{\lambda}\right\} .
$$

Entropy measure is an important information measure for fuzzy set. Entropy measures have wide applications in various fields, such as pattern recognition, clustering analysis, approximate reasoning, image processing, and decision making [16-19]. But little research has been done about hesitant fuzzy entropy measure. To construct a new hesitant fuzzy entropy measure, we firstly recall the axiomatic definition of entropy for HFEs which firstly given by $\mathrm{Xu}$ and Xia [14].

Definition 3 [14]. An entropy on HFE $\alpha$ is a real-valued function $E: H \rightarrow[0,1]$, satisfying the following axiomatic requirements:

(P1) $E(\alpha)=0$, if and only if $\alpha=0$ or $\alpha=1$

(P2) $E(\alpha)=1$, if and only if $\alpha_{\sigma(j)}+\alpha_{\sigma(l-j+1)}=1$, for $j=1,2, \ldots, l$ 
(P3) $E(\alpha) \leq E(\beta)$, if (i) $\alpha_{\sigma(j)} \leq \beta_{\sigma(j)}$ for $\beta_{\sigma(j)}+\beta_{\sigma(l-j+1)} \leq 1$ or (ii) $\alpha_{\sigma(j)} \geq \beta_{\sigma(j)}$, for $\beta_{\sigma(j)}+\beta_{\sigma(l-j+1)} \geq 1, j=1,2, \ldots, l$;

(P4) $E(\alpha)=E\left(\alpha^{c}\right)$.

Motivated by the entropy measures for fuzzy sets, we can construct some entropy formulas based on Definition 3 as follows:

$$
E(\alpha)=\frac{1}{l} \sum_{j=1}^{l} \frac{2 \alpha_{\sigma(j)}\left(1-\alpha_{\sigma(l-j+1)}\right)}{\alpha_{\sigma(j)}^{2}+\left(1-\alpha_{\sigma(l-j+1)}\right)^{2}}
$$

Theorem 1 Let $\alpha$ be a HFE, then the above measure $E(\alpha)$ is hesitant fuzzy entropy.

Proof. Obviously, $E(\alpha) \in[0,1]$, then in the following discussion, we will prove the measure $E(\alpha)$ satisfies the axiomatic requirements in definition 3 .

(P1) Obviously, when $\alpha=0$ or $\alpha=1, E(\alpha)=0$.

Now, suppose $E(\alpha)=0$, then we have $\alpha_{\sigma(j)}\left(1-\alpha_{\sigma(l-j+1)}\right)=0$,

Hence, $\alpha_{\sigma(j)}=0$ or $\alpha_{\sigma(l-j+1)}=1, j=1,2, \ldots, l$.

(P2) If $\alpha_{\sigma(j)}+\alpha_{\sigma(l-j+1)}=1$, for $j=1,2, \ldots, l$, it is easily to see that $E(\alpha)=1$.

Now, suppose $E(\alpha)=1$, then we have $\frac{2 \alpha_{\sigma(j)}\left(1-\alpha_{\sigma(l-j+1)}\right)}{\alpha_{\sigma(j)}^{2}+\left(1-\alpha_{\sigma(l-j+1)}\right)^{2}}=1$,

which is equivalently with $\alpha_{\sigma(j)}+\alpha_{\sigma(l-j+1)}=1$, for $j=1,2, \ldots, l$.

(P3) Construct function $f(x, y)$ as

$f(x, y)=\frac{2 x(1-y)}{x^{2}+(1-y)^{2}}$, where $x, y \in[0,1]$

Taking the partial derivative of $f(x, y)$ with respect to $\mathrm{x}$ and $\mathrm{y}$, respectively, yields

$$
\frac{\partial f(x, y)}{\partial x}=\frac{2(1-y)(1-y+x)(1-y-x)}{\left[x^{2}+(1-y)^{2}\right]^{2}}
$$

and

$$
\frac{\partial f(x, y)}{\partial y}=\frac{2 x(1-y+x)(1-y-x)}{\left[x^{2}+(1-y)^{2}\right]^{2}}
$$

By Equation (2) and Equation (3), when $x, y \in[0,1]$, and $x+y \in[0,1]$, we have $\frac{\partial f(x, y)}{\partial x} \geq 0, \frac{\partial f(x, y)}{\partial y} \geq 0$. Then the function $f(x, y)$ is increasing with respect to $\mathrm{x}$ and $\mathrm{y}$ ,thus if in case(i), we have $f\left(\alpha_{\sigma(j)}, \alpha_{\sigma(l-j+1)}\right) \leq f\left(\beta_{\sigma(j)}, \beta_{\sigma(l-j+1)}\right)$, then it is easily to show $E(\alpha) \leq E(\beta)$.

Similarly, when $x, y \in[0,1]$, and $x+y \in[1,+\infty)$, we have $\frac{\partial f(x, y)}{\partial x} \leq 0, \frac{\partial f(x, y)}{\partial y} \leq 0$, thus if in case(ii), we have $f\left(\alpha_{\sigma(j)}, \alpha_{\sigma(l-j+1)}\right) \leq f\left(\beta_{\sigma(j)}, \beta_{\sigma(l-j+1)}\right)$, then it is also easily to show $E(\alpha) \leq E(\beta)$.

(P4) Because $\alpha^{c}=\left\{1-\alpha_{\sigma(1)}, 1-\alpha_{\sigma(2)}, \ldots, 1-\alpha_{\sigma(l)}\right\}$, then

$$
E\left(\alpha^{c}\right)=\frac{1}{l} \sum_{j=1}^{l} \frac{2\left(1-\alpha_{\sigma(j)}\right) \alpha_{\sigma(l-j+1)}}{\left(1-\alpha_{\sigma(j)}\right)^{2}+\alpha_{\sigma(l-j+1)}^{2}}=\frac{1}{l} \sum_{k=1}^{l} \frac{2\left(1-\alpha_{\sigma(l-k+1)}\right) \alpha_{\sigma(k)}}{\left(1-\alpha_{\sigma(l-k+1)}\right)^{2}+\alpha_{\sigma(k)}^{2}}=E(\alpha) .
$$

Definition 4[12]. Let $h_{1}$ and $h_{2}$ be two HFSs on $X=\left\{x_{1}, x_{2}, \cdots, x_{n}\right\}$, then the hesitant normalized Euclid distance measure between $h_{1}$ and $h_{2}$ is defined as: 


$$
\left\|h_{1}-h_{2}\right\|=\sqrt{\frac{1}{l} \sum_{i=1}^{l}\left(h_{1}^{\sigma(j)}-h_{2}^{\sigma(j)}\right)^{2}}
$$

where $l(h)$ is the number of the elements in the $h$, in most cases, $l\left(h_{1}\right) \neq l\left(h_{2}\right)$, and for convenience, let $l=\max \left\{l\left(h_{1}\right), l\left(h_{2}\right)\right\}$. If $l\left(h_{1}\right)>l\left(h_{2}\right)$, then to operate correctly, we need to add some values to the HFE $h_{2}$. The added values mainly depend on the decision makers' risk preferences. Optimists anticipate desirable outcomes and may add the maximum value, while pessimists expect unfavorable outcomes and may add the minimum value. For example, let $h_{1}=\{0.1,0.2,0.3\}, h_{2}=\{0.4,0.5\}$.According to above analysis, optimists will extend $h_{2}$ to $h_{2}=\{0.4,0.5,0.5\}$, and pessimists will extend $h_{2}$ to $h_{2}=\{0.4,0.4,0.5\}$.Although the results may be different if we extend the shorter one by adding different values, this is reasonable because the decision makers' risk preferences can directly influence the final decision(Liu and Wang[20]; Merigó and Gil-Lafuente[21]; Zhang and Wei[12] ).

In this paper, we assume that the decision makers are all pessimistic (other situations can be studied similarly).

\section{New Decision Making Method}

There are already many multi-attribute decision-making (MADM) method are developed under different environments, which are the attribute values expressed crisp numbers, interval numbers, triangular fuzzy numbers and intuitionistic fuzzy numbers. In the decision-making process, sometimes, the information about attribute weights is completely unknown because of time pressure, lack of knowledge or data, and the expert's limited expertise about the problem domain (Chou et al.[22]; Xia and Xu [23]). In this section, we shall we extend the TOPSIS method to solve MADM problem with the hesitant fuzzy set information based an extension entropy method with the new hesitant fuzzy entropy measure. The calculation process of the hesitant fuzzy MADM method is shown in the Figure 1. 


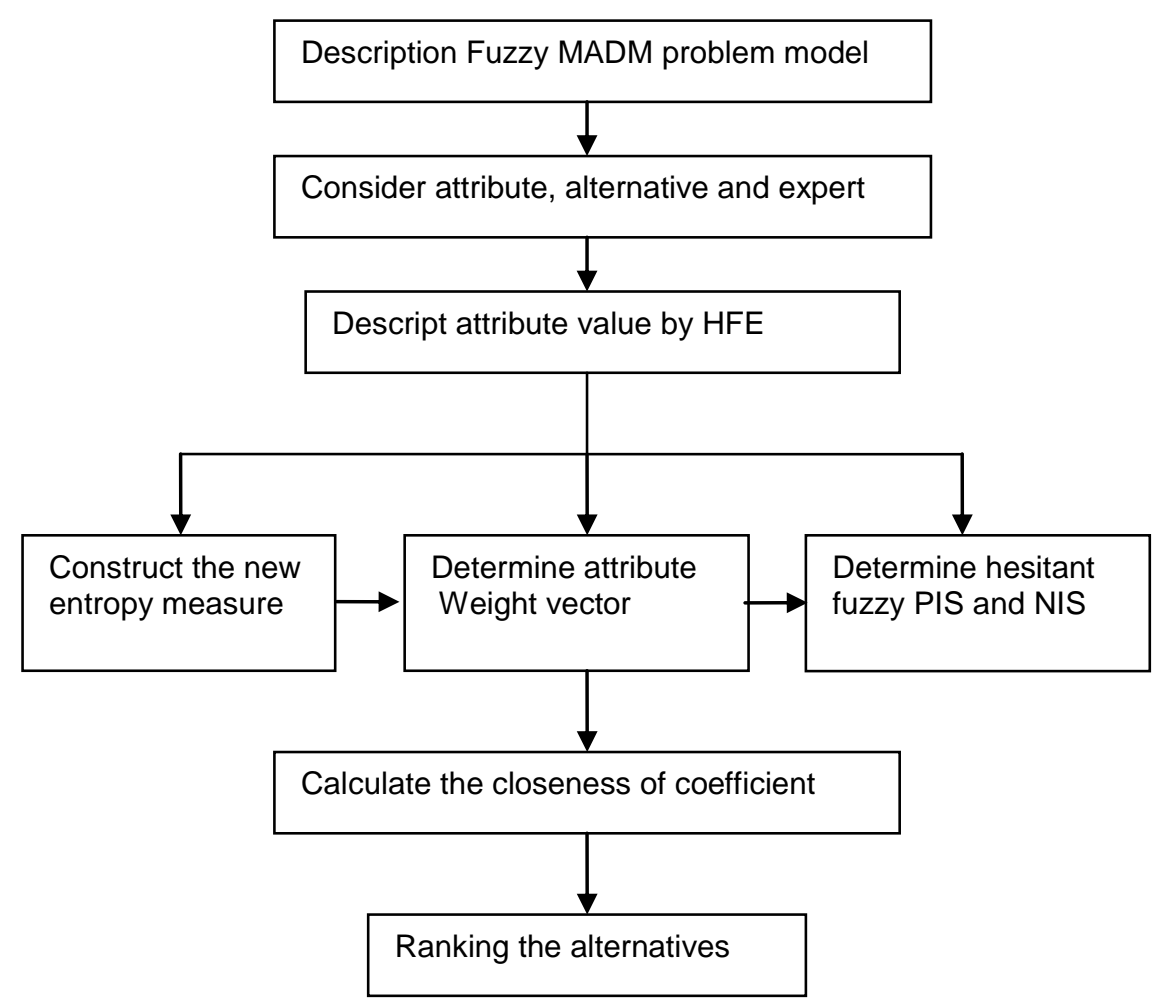

Figure 1. The Calculation Process of the Hesitant Fuzzy MADM

For a MADM problem, there are $m$ alternatives $A_{1}, A_{2}, \cdots, A_{m}$, and $n$ attributes $G_{1}, G_{2}, \cdots, G_{n}$. Suppose that the attribute weight vector $w=\left(w_{1}, w_{2}, \ldots, w_{n}\right)^{T}$ satisfies $w_{j} \in[0,1], j=1,2, \ldots, n$ and $\sum_{j=1}^{n} w_{j}=1$. Based on the above analysis, we give the new decision making methods as follows:

Step 1. The decision-maker provides all the possible evaluations about the alternative $A_{i}$ satisfies the attribute $G_{j}$, denoted by an HFE $h_{i j}$. Then we get the following decision matrix

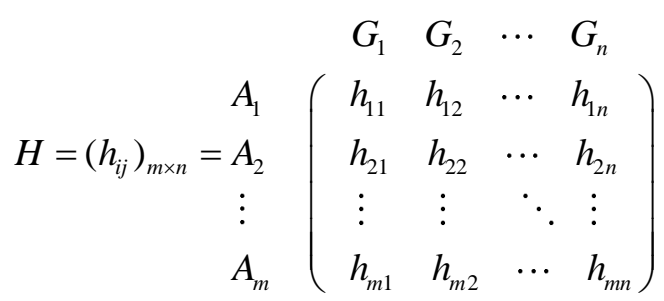

Step 2. If the information about the weight $w_{j}$ of the attribute $G_{j}$ is unknown completely, then we use the entropy weight method to determine the attribute weights. And the weight of $j$ th attribute is defined as follows:

$$
w_{j}=\frac{1-e_{j}}{n-\sum_{j=1}^{n} e_{j}}, \quad j=1,2, \ldots, n
$$


Here $e_{j}=\frac{1}{m} \sum_{i=1}^{m} E\left(h_{i j}\right), \quad j=1,2, \ldots, n$, and each $E\left(h_{i j}\right)$ is calculated by Equation (1).

Step 3. Let $I$ and $J$ be the sets of benefit attributes and cost attributes, respectively. Define the hesitant fuzzy positive ideal solution (PIS) is

$$
A^{*}=\left\{<x_{1}, h_{1}^{+}>,<x_{2}, h_{2}^{+}>, \cdots,<x_{n}, h_{n}^{+}>\right\}
$$

Where $<x_{j}, h_{j}^{+}>=<x_{j},\left\{\left(h_{j}^{1}\right)^{+},\left(h_{j}^{2}\right)^{+}, \cdots,\left(h_{j}^{l}\right)^{+}\right\}>, \quad\left(h_{j}^{k}\right)^{+}=\left\{\begin{array}{l}\max _{i=1, \cdots, m}\left\{h_{i j}^{\sigma(k)}\right\}, j \in I \\ \min _{i=1, \cdots, m}\left\{h_{i j}^{\sigma(k)}\right\}, j \in J\end{array}\right.$ and $k=1,2, \ldots, l ;$

And the hesitant fuzzy negative ideal solution (NIS) is

$A^{*}=\left\{<x_{1}, h_{1}^{-}>,<x_{2}, h_{2}^{-}>, \cdots,<x_{n}, h_{n}^{-}>\right\}$,

where $<x_{j}, h_{j}^{-}>=<x_{j},\left\{\left(h_{j}^{1}\right)^{-},\left(h_{j}^{2}\right)^{-}, \cdots,\left(h_{j}^{l}\right)^{-}\right\}>, \quad\left(h_{j}^{k}\right)^{-}=\left\{\begin{array}{l}\min _{i=1, \cdots, m}\left\{h_{i j}^{\sigma(k)}\right\}, j \in I \\ \max _{i=1, \cdots, m}\left\{h_{i j}^{\sigma(k)}\right\}, j \in I\end{array}\right.$ and $k=1,2, \ldots, l$.

Step 4.Calculate the distance measure between the alternative $A_{i}$ and the positive ideal solution (PIS) or the negative-ideal solution (NIS) as follows:

$$
d_{i}\left(A_{i}, A^{*}\right)=\sum_{j=1}^{n} w_{j} d\left(h_{i j}, h_{j}^{*}\right)
$$

and

$$
d_{i}\left(A_{i}, A^{-}\right)=\sum_{j=1}^{n} w_{j} d\left(h_{i j}, h_{j}^{-}\right)
$$

Here the distance measure $d\left(h_{i j}, \cdot\right)$ is defined in Definition 4.

Step 5.Calculate the closeness of coefficient of each alternative $A_{i}$ to the ideal solution, which defined as follows:

$$
C\left(A_{i}\right)=\frac{d_{i}\left(A_{i}, A^{-}\right)}{d_{i}\left(A_{i}, A^{*}\right)+d_{i}\left(A_{i}, A^{-}\right)}
$$

Step 6.Rank the alternatives $A_{i}(\mathrm{i}=1,2, \ldots, m)$ according to the values of $C\left(A_{i}\right)(\mathrm{i}=1,2, \ldots, m)$ by the following rule:

The smaller the value of $C\left(A_{i}\right)$ is, the better the alternative $A_{i}$ is.

\section{Case Analysis}

To illustrate effectiveness and practicability of the proposed method, we adapted an example from Boran et al. [24] and Xu and Xia [14]. An automotive company is desired to select the most appropriate supplier for one of the key elements in its manufacturing process. After pre-evaluation, four suppliers are selected as the candidate alternatives for further evaluation. To evaluate alternative suppliers, four attributes are considered as $G_{1}$ : Product quality, $G_{2}$ : Relationship closeness, $G_{3}$ : Delivery performance, and $G_{4}$ : Price. Here, $G_{1}, G_{2}$, and $G_{3}$ are the benefit attributes, and $G_{4}$ is the cost attribute. The decision maker provides all the possible assessments of the alternative $A_{i}$ on the attribute $G_{j}$, For example, to evaluate the degrees that the alternative $A_{1}$ should satisfy the attribute $G_{1}$, some experts assign 0.2 , some assign 0.4 and the others assign 0.7 , and these three parts cannot persuade each other, therefore, the degrees that the alternative $A_{1}$ satisfy the 
attribute $G_{1}$ can be considered as an HFE $h_{i j}$ and noted as $\{0.2,0.4,0.7\}$. The hesitant fuzzy decision matrix $H=\left(h_{i j}\right)_{m \times n}$ is shown in Table 1.

Table 1. Hestiant Fuzzy Decision Matrix

\begin{tabular}{ccccc}
\hline & $G_{1}$ & $G_{2}$ & $G_{3}$ & $G_{4}$ \\
\hline$A_{1}$ & $\{0.2,0.4,0.7\}$ & $\{0.1,0.2,0.5,0.7\}$ & $\{0.2,0.3,0.5,0.7,0.8\}$ & $\{0.1,0.4,0.6\}$ \\
$A_{2}$ & $\{0.4,0.6,0.7\}$ & $\{0.1,0.2,0.4,0.6\}$ & $\{0.3,0.4,0.6,0.8,0.9\}$ & $\{0.1,0.2,0.4\}$ \\
$A_{3}$ & $\{0.2,0.3,0.6\}$ & $\{0.3,0.4,0.5,0.9\}$ & $\{0.2,0.4,0.6,0.7,0.8\}$ & $\{0.3,0.4,0.8\}$ \\
$A_{4}$ & $\{0.2,0.3,0.5\}$ & $\{0.2,0.3,0.5,0.7\}$ & $\{0.4,0.6,0.7,0.8,0.9\}$ & $\{0.1,0.2,0.7\}$ \\
\hline
\end{tabular}

Suppose that the information about the attribute weights is unknown completely, to get the optimal alternative, we use the proposed method to solve it and the calculation steps are given as follows:

Step 1.Calculate the entropy matrix by Equation (1) and the results are reported in Table 2:

Table 2. Entropy Matrix

\begin{tabular}{ccccc}
\hline & $C_{1}$ & $C_{2}$ & $C_{3}$ & $C_{4}$ \\
\hline$Y_{1}$ & 0.2867 & 0.2900 & 0.3020 & 0.2733 \\
$Y_{2}$ & 0.2600 & 0.2550 & 0.2920 & 0.1933 \\
$Y_{3}$ & 0.2567 & 0.2900 & 0.2920 & 0.2867 \\
$Y_{4}$ & 0.2367 & 0.2800 & 0.2460 & 0.2733 \\
\hline
\end{tabular}

Then by Equation (6), the attribute weight vector can be obtained as: $w=\left(w_{1}, w_{2}, \ldots, w_{n}\right)^{T}=(0.2533,0.2469,0.2454,0.2544)^{T}$.

Step 2. Utilize Equation (9) and Equation (10) to calculate the Euclid distance between the alternative $A_{i}$ and the PIS or the NIS:

$$
\begin{aligned}
& d_{1}\left(A_{1}, A^{*}\right)=0.1735, d_{2}\left(A_{2}, A^{*}\right)=0.0793, d_{1}\left(A_{1}, A^{*}\right)=0.1631, d_{4}\left(A_{4}, A^{*}\right)=0.1346 \\
& \text { and } \\
& d_{1}\left(A_{1}, A^{-}\right)=0.0917, d_{2}\left(A_{2}, A^{-}\right)=0.1568, d_{3}\left(A_{3}, A^{-}\right)=0.0825, d_{4}\left(A_{4}, A^{-}\right)=0.1166 .
\end{aligned}
$$

Step 3.Calculate the closeness of coefficient of each alternative $A_{i}$ to the ideal solution by Equation (11):

$C\left(A_{1}\right)=0.3458, C\left(A_{2}\right)=0.6643, C\left(A_{3}\right)=0.3360, C\left(A_{4}\right)=0.4642$.

Step 4. Rank all the alternatives $A_{i}(i=1,2,3,4)$ according to the values of $C\left(A_{i}\right)(i=$ $1,2,3,4)$ in ascending order, then the ranking order is obtained as: $A_{2}>A_{4}>A_{3}>A_{1}$. This result is also in agreement with the one obtained in (Xu and Xia [14]).

\section{Conclusion}

Though many information measures have been developed for hesitant fuzzy sets, still there is a scope that better measures can be developed, which will find applications in a variety of fields. In this paper, we have proposed a new information entropy measure for hesitant fuzzy sets. The measure is a complement of existing entropy measure for hesitant fuzzy sets. Furthermore, based on the new entropy measure, a decision making method is put forward for the multi-attribute decision making problem, in which the attribute values expressed with HFEs. Finally, an example is given to show that the new decision making method is practical and effective. The proposed method can also be extended to other multi-attribute decision making problems, such as machine selection, supplier evaluation, in which attribute values are expressed with hesitant fuzzy numbers. 


\section{References}

[1] L. A. Zadeh, "Fuzzy sets", Information and Control, vol. 8, no. 3, (1965), pp. 338-353.

[2] K. T. Atanassov, "Intuitionistic fuzzy sets", Fuzzy Sets and Systems, vol. 20, no. 1, (1986), pp. 87-96.

[3] K. T. Atanassov and G. Gargov, "Interval-valued intuitionistic fuzzy sets", Fuzzy Sets and Systems, vol. 31, no. 3, (1989), pp. 343-349.

[4] Z. S. Xu, "Intuitionistic preference relations and their application in group decision making", Information Science, vol. 177, no. 11, (2007), pp. 2363-2379.

[5] D. F. Li, "Multiattribute decision making method based on generalized OWA operators with intuitionistic fuzzy sets", Expert Systems with Applications, vol. 37, (2010), pp. 8673-8678.

[6] G. W. Wei, "Some induced geometric aggregation operators with intuitionistic fuzzy information and their application to group decision making", Applied Soft Computing, vol. 10, no. 2, (2010), pp. 423431.

[7] V. Torra and Y. Narukawa, "On hesitant fuzzy sets and decision", The 18th IEEE International Conference on Fuzzy Systems, Jeju Island, Korea, (2009) August 20-24.

[8] V. Torra, "Hesitant fuzzy sets", International Journal of Intelligent Systems, vol. 25, (2010), pp. 529539.

[9] Z. S. Xu and M. M. Xia, "On distance and correlation measures of hesitant fuzzy information", International Journal of Intelligent Systems, vol. 26, (2011), pp. 410-425.

[10] M. M. Xia and Z. S. Xu, "Hesitant fuzzy information aggregation in decision making", International Journal of Approximate Reasoning, vol. 52, no. 3, (2011), pp. 395-407.

[11] Z. S. Xu and X. L. Zhang, "Hesitant fuzzy multi-attribute decision making based on TOPSIS with incomplete weight information", Knowledge-Based Systems, vol. 52, (2013), pp. 53-64

[12] N. Zhang and G. W. Wei, "Extension of VIKOR method for decision making problem based on hesitant fuzzy set”, Applied Mathematical Modelling, vol. 37, no. 7, (2013), pp. 4938-4947.

[13] Z. S. Xu and M. M. Xia, "Distance and similarity measures for hesitant fuzzy sets", Information Sciences, vol. 181, (2011), pp. 2128-2138.

[14] Z. S. Xu and M. M. Xia, "Hesitant fuzzy entropy and cross-entropy and their use in multiattribute decision-making", International Journal of Intelligent Systems, vol. 27, (2012), pp. 799-822.

[15] B. Farhadinia, "Information measures for hesitant fuzzy sets and interval-valued hesitant fuzzy sets", Information Sciences, vol. 240, (2013), pp. 129-144.

[16] M. S. Yang and D. C. Lin, "On similarity and inclusion measures between type-2 fuzzy sets with an application to clustering”, Computers \& Mathematics with Applications, vol. 57, (2009), pp. 896-907.

[17] T. J. Wang, Z. D. Lu and F. Li, "Bidirectional approximate reasoning based on weighted similarity measures of vague sets", Computer Engineering \& Science, vol. 24, (2002), pp. 96-100.

[18] S. K. Pal and R. A. King, "Image enhancement using smoothing with fuzzy sets", IEEE Transactions on Systems, Man and Cybernetics, vol. 11, (1981), pp. 495-501.

[19] J. Ye, "Multicriteria fuzzy decision-making method using entropy weights-based correlation coefficients of interval-valued intuitionistic fuzzy sets", Applied Mathematical Modelling, vol. 34, no. 12, (2010), pp. 3864-3870.

[20] H. W. Liu and G. J. Wang, "Multi-criteria decision-making methods based on intuitionistic fuzzy sets", European Journal of Operational Research, vol. 179, (2007), pp. 220-233.

[21] J. M. Merigó and A. M. Gil-Lafuente, "The induced generalized OWA operator", Information Sciences, vol. 179, (2009), pp. 729-741.

[22] S. Y. Chou, Y. H. Chang and C. Y. Shen, "A fuzzy simple additive weighting system under group decision-making for facility location selection with objective/subjective attributes", European Journal of Operational Research, vol. 189, (2008), pp. 132-145.

[23] M. M. Xia and Z. S. Xu, "Entropy/cross-entropy based group decision making under intuitionistic fuzzy environment", Information Fusion, vol. 13, (2012), pp. 31-47.

[24] F. E. Boran, S. Genc, M. Kurt and D. Akay, "A multi-criteria intuitionistic fuzzy group decision making for supplier selection with TOPSIS method”, Expert Systems with Applications, vol. 36, no. 8, (2009), pp. 11363-11368.

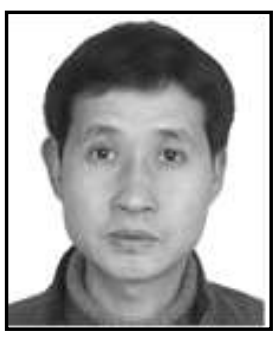

Zhengkun Yin, he is a lecture of Changsha Vocational \& Technical College. He obtained his bachelor degree of Physics in 1994 from Hunan Normal University. He obtained his master degree of computer science in 2012 from Central South University. His major researches are mainly fuzzy multi-attribute decision making and information fusion. 